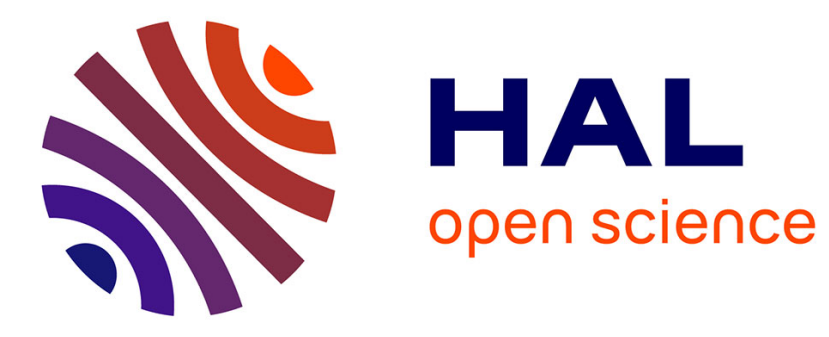

\title{
Cooperative human-robot haptic navigation
}

\author{
Stefano Scheggi, Marco Aggravi, Fabio Morbidi, Domenico Prattichizzo
}

\section{To cite this version:}

Stefano Scheggi, Marco Aggravi, Fabio Morbidi, Domenico Prattichizzo. Cooperative human-robot haptic navigation. ICRA 2014 - IEEE International Conference on Robotics and Automation, May 2014, Hong Kong, China. pp.2693-2698. hal-00979696

\section{HAL Id: hal-00979696 https://hal.science/hal-00979696}

Submitted on 16 Apr 2014

HAL is a multi-disciplinary open access archive for the deposit and dissemination of scientific research documents, whether they are published or not. The documents may come from teaching and research institutions in France or abroad, or from public or private research centers.
L'archive ouverte pluridisciplinaire HAL, est destinée au dépôt et à la diffusion de documents scientifiques de niveau recherche, publiés ou non, émanant des établissements d'enseignement et de recherche français ou étrangers, des laboratoires publics ou privés. 


\title{
Cooperative human-robot haptic navigation
}

\author{
S. Scheggi, M. Aggravi, F. Morbidi, D. Prattichizzo
}

\begin{abstract}
This paper proposes a novel use of haptic feedback for human navigation with a mobile robot. Assuming that a path-planner has provided a mobile robot with an obstaclefree trajectory, the vehicle must steer the human from an initial to a desired target position by only interacting with him/her via a custom-designed vibro-tactile bracelet. The subject is free to decide his/her own pace and a warning vibrational signal is generated by the bracelet only when a large deviation with respect to the planned trajectory is detected by the vision sensor on-board the robot. This leads to a cooperative navigation system that is less intrusive, more flexible and easy-to-use than the ones existing in literature. The effectiveness of the proposed system is demonstrated via extensive real-world experiments.
\end{abstract}

\section{INTRODUCTION}

\section{A. Motivation and related work}

Let us assume that a human wants to reach a final location in a large environment with the help of a mobile robot (see Fig. 1). Possible scenarios include assistance of an older adult or a visually-impaired person, or helping a person who is in a dangerous situation with poor visibility and no way of hearing clearly due to environmental noise.

The human is free to select his/her desired linear velocity and the robot should not force him/her to its pace as long as environmental obstacles are avoided and he/she is able to safely reach the target location. The robot guides the human by only regulating his/her angular velocity: a simple and lowintrusive way to do this is to use haptic signals provided by wearable devices (e.g., a wrist-worn bracelet). In this way, the person always remains in charge of the final decision to take, and he/she can always override the "suggestions" given by the navigation system. The type of correction made by the haptic bracelet should always be perceived as very soft, and unnatural vibrations should be avoided as much as possible.

The main source of inspiration for this work came from [1], where a passive approach inspired by the classical "Cobot" philosophy [2] is adopted for guiding an elderly person using the brakes of a commercial walker, and from [3] where the authors propose a leader-follower formation control strategy, which in this paper has been adapted to our specific human-robot setup.

A large body of literature exists, indeed, on the theme of assistive robotics and human-robot cooperation/navigation. In [4], an EKF-based indoor localization system is proposed

The research leading to these results has received funding from the European Union Seventh Framework Programme FP7/2007-2013 under grant agreement n. 601165 of the project "WEARHAP - WEARable HAPtics for humans and robots" and under grant agreement n. 288917 of the project "DALi - Devices for Assisted Living".

S. Scheggi, M. Aggravi and D. Prattichizzo are with Department of Information Engineering and Mathematics, University of Siena, 53100 Siena, Italy. D. Prattichizzo is also with the Department of Advanced Robotics, Istituto Italiano di Tecnologia, Genova, 16163, Italy \{scheggi, aggravi, prattichizzo\}edii.unisi.it

F. Morbidi is with the NeCS team, INRIA Grenoble Rhône-Alpes, 38334 Montbonnot Saint Martin, France fabio.morbidi@inria.fr

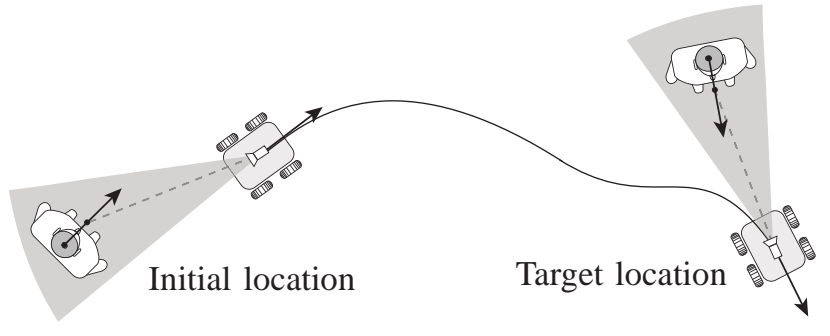

Fig. 1. Cooperative human-robot navigation from an initial to a target location (top view): the robot and human are respectively equipped with a vision sensor and a wrist-worn vibro-tactile bracelet. The field of view of the vision sensor (an RGB-D camera in this study) is shaded.

for the visually impaired. The sensing platform consists here of an inertial measurement unit and a 2D laser scanner. In [5], the authors proposed an approach that exploits the arm compliance of a humanoid robot to follow the human guidance in a physical cooperative task. The robot estimates the human pose and moves accordingly. In [6], the authors developed an assistive-guide prototype robot to help visuallyimpaired people to navigate unfamiliar areas. However the control of the robot does not account for the user's motion but only considers the deviation of the robot from the reference path or the proximity to obstacles. Moreover, the authors did not focus on the human-robot interaction. In [7], the authors proposed a travel aid for the blind which consists of a belt, a portable computer and ultrasonic sensors. Acoustic signals displayed to the user via stereophonic headphones allow to guide him/her around obstacles in pursuit of the target direction as well as to represent an image of the environment using stereophonic effects.

Noninvasive human-robot interaction can be easily achieved via haptic feedback. In fact, visual and auditory channels may be overloaded with information, thus resulting in a rapid error increase and in a consequent reduction in overall user performance. A possible solution to this problem is to deliver this information through an underutilized sensory channel. As with sound, a tactile stimulus is made up of a signal with varying frequency and amplitude, but differently from the auditory feedback, tactile feedback directly engages our motor learning system [8]. In [9], the authors applied force and position signals to the user's hand in order to guide the human operator in large environments. The position of the user is transformed into the target environment. A desired target path is then inferred based on the human's motion, on additional information from the target environment, or on task-specific criteria. Similar approaches usually focus on tele-operating a remote device and guiding the user in the remote environment. However, although haptic devices which provide kinesthetic feedback generate strong 
forces and effectively guide human motion, they are typically bulky, grounded and not portable in large environments. To address this issue, in [10], an haptic belt is used for waypoint navigation: however, the system relies not only on vibro-tactile stimuli but also on GPS information which is not available indoors or in some outdoor environments (e.g. urban areas with tall buildings).

\section{B. Original contributions and organization}

The setup considered in this paper consists of a mobile robot equipped with a vision sensor (an RGB-D camera) and a human subject wearing a custom-designed vibro-tactile bracelet. The robot follows a pre-planned path and helps the human to reach a desired target position. A predefined distance and orientation should be maintained between the human and the robot at all times. The leader-follower formation control strategy proposed in [3] has been tailored to our human-robot setup: in fact, recent studies [11] have shown a close relationship between the shape of human locomotor paths in goal-directed movements, and the simplified kinematic model of a wheeled mobile robot. Thus, nonholonomic constraints similar to those governing the motion of mobile robots, seem to be at work when a human is walking: in particular, the authors have shown that the shoulders can be considered as a sort of steering wheel that drives the human body with a short delay (of about one fifth of a second). Differently from [3], in our scenario the human should always be able to freely select his/her linear velocity. A specific haptic feedback is sent to the user in order to adjust his/her angular velocity according to the formation specifics. Our purpose is to send easily processable on/off signals to the human, so that he/she can promptly respond to the stimuli of the guiding robot.

The rest of the paper is organized as follows. Sect. II and Sect. III present our control strategy and the haptic-feedback generation mechanism, respectively. Sect. IV describes our human visual detection algorithm and Sect. V reports the results of real-world experiments. Finally, in Sect. VI conclusions are drawn and possible subjects of future research are outlined.

\section{PROBlem FORMULATION}

In this section, we briefly review the leader-follower formation control strategy proposed in [3], and show how to adapt it to our human-guidance problem.

\section{A. Formation control setup}

Consider a robot modeled as a unicycle,

$$
\dot{x}=v \cos \theta, \quad \dot{y}=v \sin \theta, \quad \dot{\theta}=\omega,
$$

where $\mathbf{R}=(x, y, \theta)^{T} \in \mathbb{R}^{2} \times \mathbb{S}^{1}$ is the pose of the robot, and $\mathbf{u}=(v, \omega)^{T}$ the control input (the linear and angular velocities). We denote by $\mathbf{P}(t)=(x(t), y(t))^{T}$ the position of the robot at time $t$ and by $\overline{\mathbf{R}}=(x(0), y(0), \theta(0))^{T}$ its initial pose.

In the leader-follower setup proposed in [3], robot $\mathbf{R}_{h}$ (in our case, a human) must follow the robot $\mathbf{R}_{r}$ with a desired distance $l^{d}$ and desired relative orientation $\psi^{d}$ (see Fig. 2). Let $\beta=\theta_{r}-\theta_{h}$ be the relative bearing of $\mathbf{R}_{h}$ and $\mathbf{R}_{r}$,

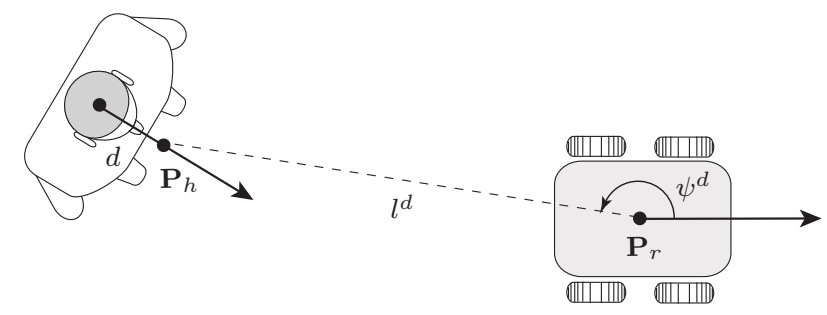

Fig. 2. Human-robot formation. $l^{d}$ and $\psi^{d}$ represent the desired distance and orientation between the human and robot, respectively.

$\mathbf{u}_{r}=\left(v_{r}, \omega_{r}\right)^{T}$ and $\mathbf{u}_{h}=\left(v_{h}, \omega_{h}\right)^{T}$ their control inputs, and

$$
\mathbf{G}=\left[\begin{array}{cc}
\cos \gamma & d \sin \gamma \\
\frac{-\sin \gamma}{l} & \frac{d \cos \gamma}{l}
\end{array}\right], \quad \mathbf{F}=\left[\begin{array}{cc}
-\cos \gamma & 0 \\
\frac{\sin \gamma}{l} & -1
\end{array}\right],
$$

where $\gamma=\beta+\psi$ and $l, \psi$ are the current relative distance and orientation of $\mathbf{R}_{h}$ and $\mathbf{R}_{r}$, and $d$ is the offset to an offaxis reference point $\mathbf{P}_{h}=\left(x_{h}, y_{h}\right)^{T}$ on $\mathbf{R}_{h}$ (see Fig. 2). The control input for $\mathbf{R}_{h}$ can then be written as,

$$
\mathbf{u}_{h}=\mathbf{G}^{-1}\left(\mathbf{q}-\mathbf{F} \mathbf{u}_{r}\right),
$$

being $\mathbf{q}$ an auxiliary control input defined as

$$
\mathbf{q}=\left[\begin{array}{c}
k_{1}\left(l^{d}-l\right) \\
k_{2}\left(\psi^{d}-\psi\right)
\end{array}\right],
$$

where $k_{1}, k_{2}$ are positive control gains (observe that $\mathbf{G}$ is always invertible as long as $d / l>0$, which is always true in practice).

Note that since nonholonomic constraints analogous to those governing the motion of a unicycle robot, seem to be at work when a human is walking [11], the control law (1) can also be applied (with suitable modifications) to mixed human-robot formations (cf. [12]). In what follows, we will show how to tailor (1) to our human-guidance problem. Notice that in our framework the distinction between leader and follower is not relevant: in fact, here both agents cooperate without direct physical interaction to achieve a common goal (reach the desired target position).

\section{B. Human-robot guidance}

Differently from [3], in our scenario the human should always be able to freely choose his/her linear velocity. However, in order to be driven by $\mathbf{R}_{r}$ to the target position, his/her angular velocity should be suitably regulated. On the other side, the robot should change its linear velocity according to that of the user, while its angular velocity depends on the specific trajectory from the initial to the target position. Since the desired geometric path of the robot is the result of an on-board planning algorithm, we assume the trajectory is smooth (i.e., the tangent to the trajectory is well defined at each point), and that its curvature is known. Typically, a planner yields a smooth trajectory which consists of the union of segments and circular arcs. The angular velocity $\omega_{r}$ of $\mathbf{R}_{r}$ that solves the path following problem, 
assuming that the initial robot configuration is not far from the desired path and that $v_{r} \neq 0$, is reported in [1]. Let

$$
\mathbf{G}^{*}=\left[\begin{array}{cc}
\frac{d \cos \gamma}{l} & \frac{\sin \gamma}{l} \\
d \sin \gamma & -\cos \gamma
\end{array}\right], \quad \mathbf{F}^{*}=\left[\begin{array}{cc}
\frac{-\sin \gamma}{l} & -1 \\
\cos \gamma & 0
\end{array}\right],
$$

then the control inputs for the human and robot are given by (cf. (1)),

$$
\left[\begin{array}{c}
\omega_{h} \\
v_{r}
\end{array}\right]=\left(\mathbf{G}^{*}\right)^{-1}\left(\mathbf{q}^{*}-\mathbf{F}^{*}\left[\begin{array}{l}
v_{h} \\
\omega_{r}
\end{array}\right]\right),
$$

where $\mathbf{q}^{*}$ is an auxiliary control input defined as

$$
\mathbf{q}^{*}=\left[\begin{array}{c}
k_{2}\left(\psi^{d}-\psi\right) \\
k_{1}\left(l^{d}-l\right)
\end{array}\right] .
$$

Note that $\operatorname{det}(\mathbf{G})=-\operatorname{det}\left(\mathbf{G}^{*}\right)$.

Assuming that the human is moving with linear velocity $v_{h}$ and that the robot is rotating with angular velocity $\omega_{r}$, the control law reported in (2) allows to maintain the formation specified by the parameters $l^{d}$ and $\psi^{d}$.

Remark 1: Suppose that the robot estimates the human motion using an on-board vision sensor with limited field of view (cf. Fig. 1 and Sect. IV). Since the formation parameters are defined in the reference frame of the robot, then a suitable choice of $l^{d}$ and $\psi^{d}$ allows to always maintain the human inside the sensor's field of view.

Note that while it is not problematic to drive a robot with velocity $v_{r}$, it is not trivial to impose a desired angular velocity $\omega_{h}$ to a human. In the next section we will show how to use haptic feedback to address this challenging problem.

\section{HAPTIC FEEDBACK}

In this section, we describe the main features of our haptic device and the nature of the vibro-tactile feedback provided to the human. We also present the results of an experimental analysis conducted to assess how the stimuli produced by the bracelet are perceived by humans.

\section{A. Description of the haptic bracelet}

Studies have demonstrated that vibration is best on hairy skin and in bony areas [13], [14]. In particular, wrists and spine are generally preferred for detecting vibrations, with arms next in line [15]. Moreover movement can decrease detection rate and increases response time of particular body areas. For example, walking affects lower body sites the most [15].

Since the haptic feedback will provide the human with information about his/her angular velocity, two vibrating motors will be used to independently warn the user. However, since real robots have strict limitations on their maximum linear velocity, a third motor needs to be used to warn the human when he/she is too close to the robot.

A bracelet shape with three vibrating motors circling the forearm, ensures sufficient distance between the motors while covering a minimal forearm area. In fact, the minimal distance between two stimuli to be differentiated is about $35 \mathrm{~mm}$ on the forearms [13], [16].

Following these guidelines, we designed a wrist-worn haptic bracelet in which three cylindrical vibro-motors, $L$ (left),

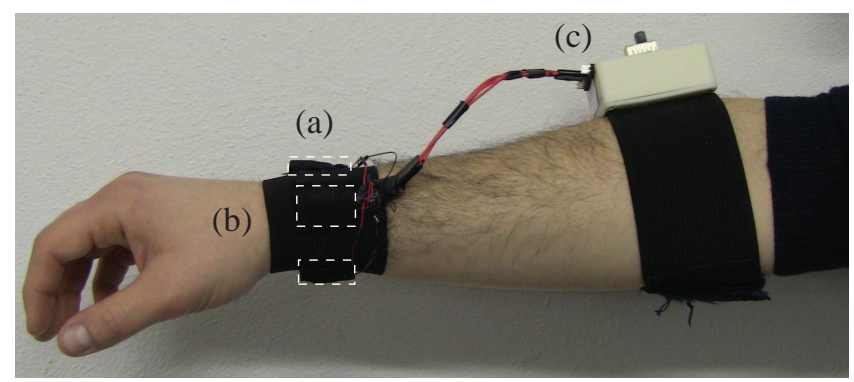

Fig. 3. Human-robot interaction is achieved via a vibro-tactile bracelet equipped with three vibrating motors (a), attached to an elastic wristband (b). The three motors are disposed equidistantly in order to improve the vibrotactile perception. The Li-Ion battery and the Arduino board are in (c).

$C$ (center) and $R$ (right) are independently controlled via an external PC using the Bluetooth communication protocol (see Fig. 3). The communication is realized with an RN42 Bluetooth module connected to an Arduino mini pro 3.3V with a baud rate of 9600. An Atmega 328 micro-controller installed on the Arduino board is used to independently control the vibration amplitude of each motor. The three Precision Microdrives 303-100 Pico Vibe $3.2 \mathrm{~mm}$ vibration motors were placed into three fabric pockets on the external surface of the bracelet (the width of the wristband is about 60 $\mathrm{mm}$ ), with shafts aligned with the elbow bone (see Fig. 3). Since the rotating masses are exposed, we placed each motor inside a plastic cylindrical case in order to protect them from damage and guarantee a correct operation. The motors have a vibration frequency range of $100 \mathrm{~Hz}-280 \mathrm{~Hz}$ (the maximal sensitivity is achieved around $200 \mathrm{~Hz}-300 \mathrm{~Hz}$ [17]).

\section{B. Haptic feedback generation}

In order to not overload the tactile channel and to not reduce the recognition time, few but significative signals should be sent to the user. To this end, we do not modulate the frequency of the signal but adopt a simple on/off mechanism. When a motor is engaged, it vibrates at a frequency of $250 \mathrm{~Hz}$ with an amplitude of $0.6 \mathrm{~g}$ in order to achieve maximal sensitivity.

Let $f_{j}(t)$ the vibration frequency of motor $j \in\{L, C, R\}$ at time $t$ and $\omega_{h}^{*}(t)$ the angular velocity (determined via (2)) that the user should have at time $t+\Delta T$ in order to properly follow the robot, where $\Delta T$ is the discrete time step. In what follows, we assume that the human velocity is constant between two consecutive frames in absence of external perturbations, i.e., $v_{h}(t+\Delta T)=v_{h}(t), \omega_{h}(t+\Delta T)=\omega_{h}(t)$.

The proposed haptic-generation mechanism consists in sending a proper vibro-tactile signal if the angular velocity $\omega_{h}^{*}(t)$ differs from the user's angular velocity $\omega_{h}(t+\Delta T)$ more than a given threshold $\alpha \in \mathbb{R}^{+}$, i.e. we set,

$$
\begin{gathered}
f_{L}(t)= \begin{cases}250 \mathrm{~Hz}, & \text { if } \omega_{h}^{*}(t)-\omega_{h}(t+\Delta T)>\alpha, \\
0 \mathrm{~Hz}, & \text { otherwise, }\end{cases} \\
f_{R}(t)= \begin{cases}250 \mathrm{~Hz}, & \text { if } \omega_{h}^{*}(t)-\omega_{h}(t+\Delta T)<-\alpha, \\
0 \mathrm{~Hz}, & \text { otherwise. }\end{cases}
\end{gathered}
$$

Since we have assumed that $\omega_{h}(t+\Delta T)=\omega_{h}(t)$, equations (3)-(4) use the angular velocity of the human at time $t$ and 


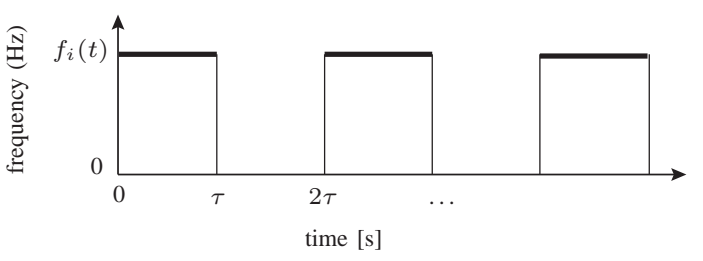

(a)

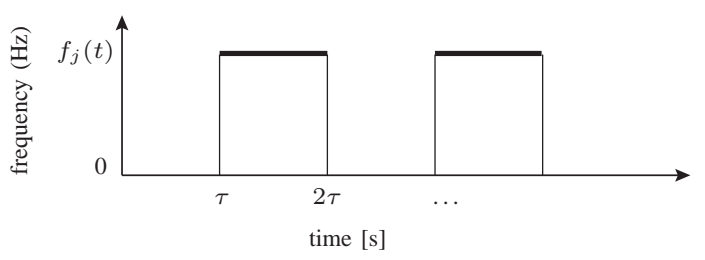

(b)

Fig. 4. Temporization of the stimuli. (a) In order to avoid the aftereffect problem, a periodic vibrational pattern with period $2 \tau$ is provided to the user. To keep signal recognition as simple as possible in the case of a combination of stimuli $f_{i}(t), f_{j}(t)(i \in\{L, R\}, j=C)$, we alternate the patterns in (a) and (b).

the output of the controller at time $t$ to generate a suitable haptic feedback in order to correct the angular velocity of the user at time $t+\Delta T$.

Since the maximal linear velocity of real-world robots is limited, the robot might be unable to maintain the formation if the human walks too fast. To avoid this, a suitable signal is sent to the central motor to warn the human when he/she is getting too close to the robot. Let $\delta \in \mathbb{R}^{+}$the minimal human-robot distance,

$$
f_{C}(t)= \begin{cases}250 \mathrm{~Hz}, & \text { if } l(t)<\delta, \\ 0 \mathrm{~Hz}, & \text { otherwise. }\end{cases}
$$

In order to reduce the aftereffect problem (vibration effects usually persist after the end of the stimulation, see [18] and the references therein), we displayed a periodic vibrational pattern with period $2 \tau$ instead of a continuous signal (see Fig. 4(a)). Moreover, to keep the signal recognition as simple as possible, in the case of a combination of stimuli, we do not superimpose them but alternate between the two patterns reported in Fig. 4(a) and 4(b), respectively. It is worth noting that we avoided cases in which all motors were turned on and cases in which the left and right motors were contemporarily activated, since they never occur in our human-guidance problem.

\section{Evaluation of the haptic bracelet}

The proposed haptic bracelet has been tested on 8 healthy subjects (6 males, age range 26-60, all right-handed): 4 of them had experience with previous prototypes of our haptic bracelet based, however, on different working principles. None of the participants reported any deficiency in the perception abilities (including vision, hearing, touch and proprioception). In the evaluation, a single signal (center $C$, left $L$, right $R$ ) or a combination of signals (center-left $C L$, center-right $C R$ ) was sent to the haptic bracelet. The subjects were asked to identify which motors were vibrating. Each participant was informed about the procedure before the beginning of the experiment and a 5-minute familiarization period was given to each subject. The evaluation consisted of two sets of 25 trials each: after each set the subjects took a small break. Every signal was proposed five times in a pseudo-randomized order with a vibrating frequency of $250 \mathrm{~Hz}$ and amplitude of $0.6 \mathrm{~g}$. Sequences of stimulation appeared in short bursts with $\tau=0.2 \mathrm{~s}$ (see Fig. 4(a)).

The subjects could correctly perceive almost the totality of the proposed stimuli (cf. Table I). The average time elapsed to perceive the stimuli was approximately $1.31 \mathrm{~s}$ with a standard deviation of $0.06 \mathrm{~s}$. In order to evaluate the statistical significance of the differences between the stimuli, we performed a one-way ANOVA [19] on the observed number of correct responses and on the elapsed time. ANOVA analyzes the groups variances to test the heterogeneity of their means. From our analysis, it turns out that the type of stimulus does not significantly influence the percentage of correct responses or the elapsed time.

\begin{tabular}{|c|c|c|c|c|c|c|}
\hline & & $\mathrm{L}$ & $\mathrm{C}$ & $\mathrm{R}$ & CL & CR \\
\hline \multirow{2}{*}{$\begin{array}{l}\text { correct } \\
\text { answers [\%] }\end{array}$} & mean & 100 & 97.5 & 100 & 95 & 97.5 \\
\hline & std & - & 6.83 & - & 11.55 & 6.83 \\
\hline \multirow{2}{*}{$\begin{array}{l}\text { response } \\
\text { time [s] }\end{array}$} & mean & 1.27 & 1.34 & 1.24 & 1.35 & 1.39 \\
\hline & std & 0.59 & 0.62 & 0.51 & 0.59 & 0.78 \\
\hline
\end{tabular}

TABLE I

EVALUATION OF THE HAPTIC BRACELET: MEAN AND STANDARD DEVIATION OF THE PERCENTAGE OF CORRECT ANSWERS AND OF RELATED RESPONSE TIME.

\section{Human VisuAl DETECTION AND TRACKING}

This section provides an overview of the major steps of our method for detecting the human from the visual information provided by an RGB-D camera on-board the robot: our approach is quite general and can be applied to other typologies of vision sensors (e.g., time-of-flight cameras) as well.

In what follows, we will assume that the robot $x y$-plane is parallel to the floor. As a preliminary step, we perform an extrinsic calibration of the RGB-D camera and robot reference frames. The homogeneous matrix $\mathbf{H}_{\mathcal{K}}^{\mathcal{R}}$, that relates the robot frame $(\mathcal{R})$ with the camera frame $(\mathcal{K})$, is estimated using the algorithm proposed in [20]. This preliminary step is necessary since the control strategy described in Sect. II is computed with respect to the robot frame. We then use NITE's skeleton tracker to detect and track the human (see Fig. 5(a)). Since the shoulders play an important role in the nonholonomic description of human locomotion (cf. Sect. I-B), in the detection phase we discard all the 3-D points that are too far from the human's torso. We first downsample the data using a voxel grid filter with a leaf size of $2 \mathrm{~cm}$ (see Fig. 5(b)). We then express the downsampled point cloud in the robot reference frame using the estimated homogeneous matrix $\mathbf{p}^{\mathcal{R}}=\mathbf{H}_{\mathcal{K}}^{\mathcal{R}} \mathbf{p}^{\mathcal{K}}$, where $\mathbf{p}^{\mathcal{K}}$ represents the point cloud of the human torso and $\mathbf{p}^{\mathcal{R}}$ the point cloud in the robot's frame. We then project $\mathbf{p}^{\mathcal{R}}$ onto the robot $x y$ plane and fit an ellipse over the points (see Fig. 5(c)). 


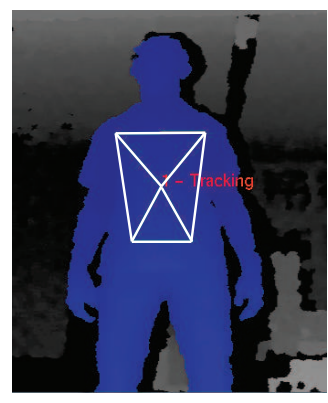

(a)

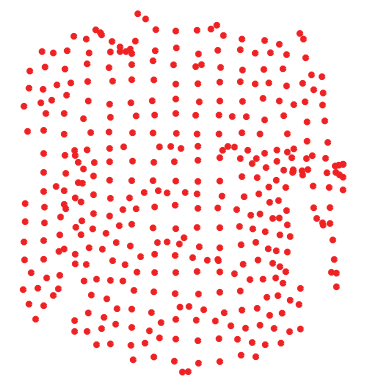

(b)

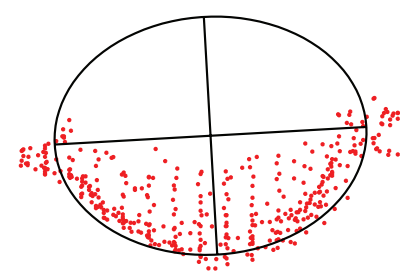

(c)

Fig. 5. Human-body tracking on real data. (a) NITE's skeleton tracker is used to track the subject (the skeleton of the torso is shown in white). (b) The points which are not close enough to the torso are discarded, while the remaining points are down-sampled and expressed in the robot's reference frame. (c) The points are finally projected onto the robot $x y$-plane and the position and orientation of the human body is determined via ellipse fitting.

To improve the robustness when the skeleton tracker fails during the human motion, we select the 3-D points in the neighborhood of the previous human pose. We project those points onto the robot $x y$-plane and then perform a cluster filtering discarding those clusters whose dimension is outside a given range and whose distance is far enough from the last tracked human position. Finally an ellipse fitting is performed over the resulting cluster. We relied on the Point Cloud Library [21] to process the 3-D point data and extract the information about the human motion.

\section{EXPERIMENTAL VALIDATION}

The effectiveness and robustness of our human-robot navigation mechanism has been tested in a large indoor environment using a Pioneer P3AT robot (which has a maximal linear velocity of $0.7 \mathrm{~m} / \mathrm{s}$ ) equipped with a backward facing Microsoft's Kinect camera.

Six healthy subjects (age range 23-30, all males and righthanded) were involved in our tests ${ }^{1}$ : five of them participated in the evaluation of the haptic bracelet (cf. Sect. III-C). All subjects were blindfolded and instructed to move according to the tactile stimulation, but no indications were given about the linear and/or angular velocities to be kept. Two different trajectories were considered for the robot in order to test the haptic feedback when the user continuously changes his direction (see Fig. 6(a)). Each subject performed 4 trials: 2 trials with trajectory $\mathrm{A}$ and 2 trials with trajectory $\mathrm{B}$ in a random order.

The visual tracking algorithm described in Sect. IV was run

\footnotetext{
${ }^{1}$ The video of the experiment is available at: http://sirslab.dii.unisi.it/research/haptic/projects/haptic-navigation/ Note that the trajectories performed in the video are different from those reported in Fig. 6.
}

at an average frame rate of $17 \mathrm{fps}$ on a laptop with $8 \mathrm{~GB}$ RAM, 2.9 GHz Intel i5 CPU and NVIDIA GeForce GT 540M (2GB DDR3) graphic card. Owing to the actuation time of the motors of the mobile robot, the control inputs of the human and robot were computed every $0.2 \mathrm{~s}$ and sent to the robot via the TCP/IP protocol. Regarding the formation parameters, we set $l^{d}=2.3 \mathrm{~m}, \psi^{d}=\pi$ and selected $k_{1}=k_{2}=5, d=0.1 \mathrm{~m}, \alpha=0.8 \mathrm{rad} / \mathrm{s}, \tau=0.2 \mathrm{~s}$ and $\delta=l^{d}-0.2 \mathrm{~m}$.

The initial and final position of the robot and human are reported in Fig. 6(a). Figs. 6(b), 6(c) show the time evolution of the Euclidean norm of the formation error

$$
\mathbf{E}(t)=\mathbf{P}_{h}(t)-l^{d}\left(\cos \left(\psi^{d}\right), \sin \left(\psi^{d}\right)\right)^{T}
$$

of the fourth subject and the related vibrational frequencies of the right, left and central motor of the haptic bracelet. Figs. 6(d), 6(e) report the mean and the standard deviation of the norm of the formation error for each of the 24 trials (i.e. 4 trials for each of the 6 subjects), and the percentage of time over the all trials in which the vibro-tactile bracelet was activated. In blue it is shown the first subject who did not participate in the evaluation of the haptic device (cf. Sect. III-C). As we can notice from Fig. 6(d), the mean of the norm of the formation error is always smaller than $0.9 \mathrm{~m}$, which we deem acceptable for the application at hand. Moreover, although the first user never tried the haptic bracelet before, he was able to correctly recognize the haptic stimuli and cooperate with the robot.

The mean of the norm of the formation error over all the trials, is $0.52 \mathrm{~m}$ with a standard deviation of $0.16 \mathrm{~m}$ and the average time in which the bracelet was turned on is the $80.9 \%$ of the total duration of the trial with a standard deviation of $10.9 \%$. In this respect, the robot trajectories represent a sort of worst case due to the continuous change of the human direction. A possible way to reduce the bracelet's activation time is to use a robot with higher maximal linear velocity, in which case the central motor of the bracelet may be eliminated. This option will be considered in our future research.

\section{CONCLUSIONS AND FUTURE WORK}

In this paper we have proposed a new haptic paradigm for the guidance of a human in an unknown environment with a mobile robot. The subject is free to decide his/her own pace and a warning vibrational signal is generated by a wrist-worn haptic bracelet only when a large deviation with respect to the planned trajectory is sensed. With respect to the existing robot navigation schemes, our cooperative guidance system is less intrusive, more flexible and user-friendly and it does not require a long training program. The effectiveness of the proposed approach is demonstrated via an extensive experimental validation. In future works, we would like to perform experiments in cluttered and noisy environments, and conduct tests with elderly people and subjects with vision or vestibular disorders.

\section{REFERENCES}

[1] D. Fontanelli, A. Giannitrapani, L. Palopoli, and D. Prattichizzo. Unicycle steering by brakes: a passive guidance support for an assistive cart. In Proc. 52nd IEEE Conf. Dec. Contr, pages 2275-2280, 2013. 


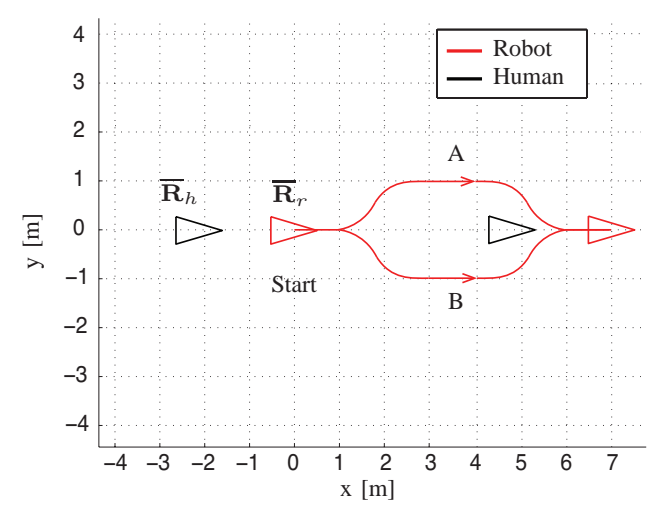

(a)

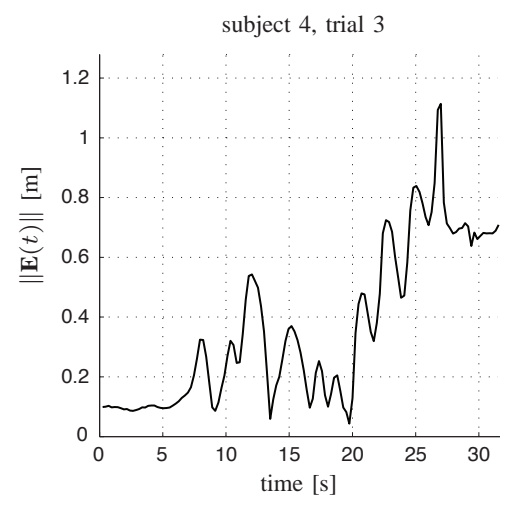

(b)
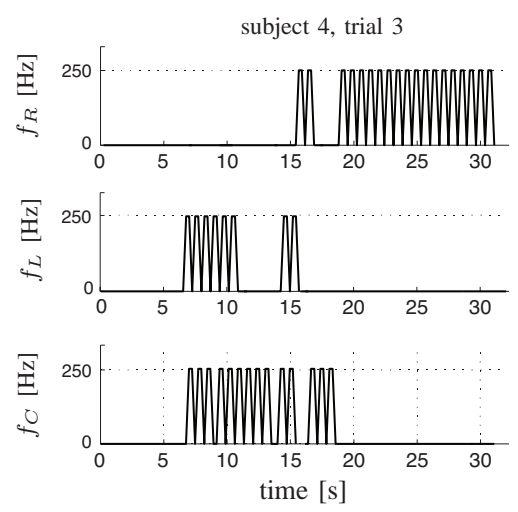

(c)

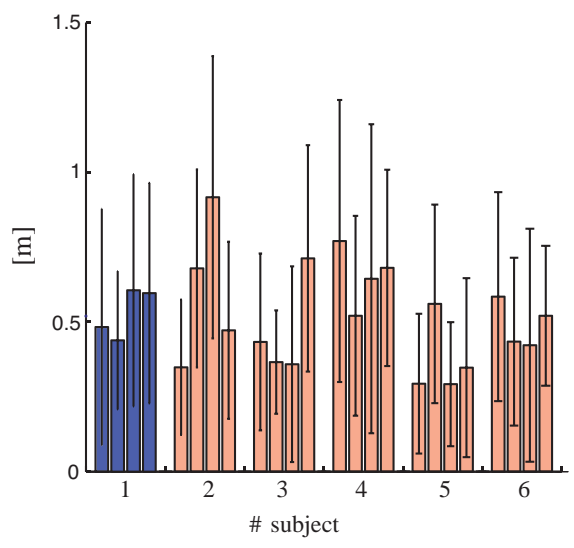

(d)
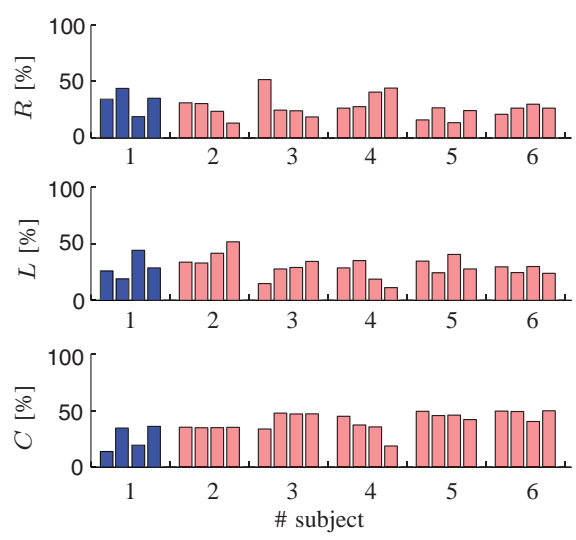

(e)

Fig. 6. Experimental results. (a) Initial and final disposition of the robot (red) and human (black) and planned trajectories of the robot (red); (b) time evolution of the Euclidean norm of the formation error $\mathbf{E}(t)=\left(E_{x}(t), E_{y}(t)\right)^{T}$ for the fourth subject and related vibrational frequencies of the right, left and central motor of the bracelet (c); (d) mean and standard deviation of the norm of the formation error over the 24 trials for the 6 subjects and (e) percentage of activation time of the motors (the first subject did not participate in the evaluation of the haptic bracelet).

[2] M. Peshkin, J. Colgate, W. Wannasuphoprasit, C. Moore, R. Gillespie, and P. Akella. Cobot architecture. IEEE Trans. Robotic. Autom., 17(4):377-390, 2001.

[3] A.K. Das, R. Fierro, V. Kumar, J.P. Ostrowski, J. Spletzer, and C.J. Taylor. A vision-based formation control framework. IEEE Trans. Robotic. Autom., 18(5):813-825, 2002.

[4] J.A. Hesch, F.M. Mirzaei, G.L. Mariottini, and S.I. Roumeliotis. A 3D pose estimator for the visually impaired. In Proc. IEEE/RSJ Int. Conf. Intel. Robots Syst., pages 2716-2723, 2009.

[5] J. Stückler and S. Behnke. Following human guidance to cooperatively carry a large object. In Proc. 11st IEEE-RAS Int. Conf. Humanoid Robots, pages 218-223, 2011.

[6] G. Galatas, C. McMurrough, G.L. Mariottini, and F. Makedon. eyeDog: an assistive-guide robot for the visually impaired. In Proc. 4th Int. Conf. PErv. Tech. Assist. Env., number 58, pages 1-8, 2011.

[7] S. Shoval, J. Borenstein, and Y. Koren. Auditory guidance with the Navbelt-a computerized travel aid for the blind. IEEE Trans. Syst. Man Cy. C, 28(3):459-467, 1998.

[8] J. Lieberman and C. Breazeal. TIKL: Development of a wearable vibrotactile feedback suit for improved human motor learning. IEEE Trans. Robot., 23(5):919-926, 2007.

[9] A.P. Arias and U.D. Hanebeck. Wide-area haptic guidance: Taking the user by the hand. In Proc. IEEE/RSJ Int. Conf. Intel. Robots Syst., pages 5824-5829, 2010.

[10] J. B. Van Erp, H. A. H. Van Veen, C. C. Jansen, and T. Dobbins. Waypoint navigation with a vibrotactile waist belt. ACM Trans. Appl. Percept., 2(2):106-117, 2005.

[11] G. Arechavaleta, J.P. Laumond, H. Hicheur, and A. Berthoz. On the nonholonomic nature of human locomotion. Auton. Robot., 25(1):25$35,2008$.
[12] S. Scheggi, F. Chinello, and D. Prattichizzo. Vibrotactile haptic feedback for human-robot interaction in leader-follower tasks. In $A C M$ PETRA Workshop on Robotics in Assistive Environments, Article No. 51, 2012.

[13] F. Gemperle, T. Hirsch, A. Goode, J. Pearce, D. Siewiorek, and A. Smailigic. Wearable vibro-tactile display. Technical report, Carnegie Mellon University, 2003.

[14] L.A. Jones and S.J. Lederman. Human hand function. Oxford University Press, 2006.

[15] I. Karuei, K.E. MacLean, Z. Foley-Fisher, R. MacKenzie, S. Koch, and M. El-Zohairy. Detecting vibrations across the body in mobile contexts. In Proc. Int. Conf. Human Factors Comp. Syst., pages 3267 $3276,2011$.

[16] S. Weinstein. Intensive and extensive aspects of tactile sensitivity as a function of body part, sex, and laterality. In Spriengfield, editor, The skin senses, pages 195-218. Erlbaum, 1968.

[17] A. Riener. Sensor-actuator supported implicit interaction in driver assistance systems. In S. Hölldobler et al., editor, Ausgezeichnete Informatikdissertationen, volume 10, pages 221-230. Gesellschaft für Informatik, Bonn, 2010.

[18] I.M. Vogels, A.M. Kappers, and J.J. Koenderink. Haptic aftereffect of curved surfaces. Perception, 25(1):109-119, 1996.

[19] R. G. Miller. Beyond ANOVA: basics of applied statistics. Chapman \& Hall, 1997

[20] Q. Zhang and R. Pless. Extrinsic calibration of a camera and laser range finder (improves camera calibration). In Proc. IEEE/RSJ Int Conf. Intel. Robots Syst., pages 2301-2306, 2004.

[21] R. B. Rusu and S. Cousins. 3D is here: Point Cloud Library (PCL). In Proc. IEEE Int. Conf. Robot. Automat, pages 1-4, 2011. 